\title{
The Measurement of P-, S-, and R-Wave Velocities to Evaluate the Condition of Reinforced and Prestressed Concrete Slabs
}

\author{
Young Hak Lee ${ }^{1}$ and Taekeun $\mathrm{Oh}^{2}$ \\ ${ }^{1}$ Department of Architecture Engineering, Kyung Hee University, 1732 Deogyeong-daero, Giheung-gu, Yongin 17104, Republic of Korea \\ ${ }^{2}$ Department of Safety Engineering, Incheon National University, 12-1 Songdo-dong, Yeonsu-gu, Incheon 406-772, Republic of Korea
}

Correspondence should be addressed to Taekeun Oh; tkoh@inu.ac.kr

Received 11 February 2016; Revised 1 July 2016; Accepted 17 July 2016

Academic Editor: Jun Liu

Copyright (C) 2016 Y. H. Lee and T. Oh. This is an open access article distributed under the Creative Commons Attribution License, which permits unrestricted use, distribution, and reproduction in any medium, provided the original work is properly cited.

\begin{abstract}
The traditional P-wave ultrasonic measurement has been used for the condition assessment of general reinforced concrete structures for a long time, but the effects of prestressing applied to concrete structures such as long-span buildings and bridges on ultrasonic pulse velocity have not been studied clearly. Therefore, this study analyzed the statistical distribution of P-wave ultrasonic pulse velocities in reinforced and prestressed concrete slabs of $3000 \times 3000 \mathrm{~mm}$ with a thickness of $250 \mathrm{~mm}$. In addition, we measured $\mathrm{S}$ - and R-waves to identify experimental consistency by statistical analysis using the Kolmogorov-Smirnov goodness-of-fit test. The experimental results show that the P-, S-, and R-wave velocities increased slightly (2-3\%) when prestressing was applied. As expected, the S- and R-wave measurements show better statistical reliability and potential for in situ evaluation than the P-wave because they are less sensitive to confinement and boundary conditions. The experimental results in this study can be used when assessing the condition of prestressed concrete structures through the velocities of elastic waves.
\end{abstract}

\section{Introduction}

The accurate measurement of concrete properties, such as elasticity, strength, and Poisson's ratio, is important in terms of the quality assurance $(\mathrm{Q} / \mathrm{A})$ and quality control $(\mathrm{Q} / \mathrm{C})$ of concrete structures [1]. The $\mathrm{Q} / \mathrm{A}$ and $\mathrm{Q} / \mathrm{C}$ require numerous tests in the laboratory. In the field, obtaining core samples consumes a lot of time, budget, and labor and can deteriorate the structure itself. Therefore, nondestructive testing is needed to eliminate problems in both the laboratory and the field.

One of the most effective nondestructive evaluation methods for the assessment of concrete structures is ultrasonic measurement [2]. It has been popularly used in both reinforced and prestressed concrete structures to determine material properties, detect defects, and assess deterioration. Recent failures of prestressed concrete structures have highlighted the need to improve inspection and maintenance in the United States and Europe. However, the field application of ultrasonic methods to prestressed concrete structures should be cautious because the effects of prestressing on the ultrasonic methods have not been clearly validated. These effects are analogous to the acoustoelastic effects that the ultrasonic wave velocity of a solid medium changes with the stress state of the medium $[3,4]$. Even though many researchers have developed the health monitoring techniques for steel structures based on the acoustoelasticity [5], only few studies have been related to reinforced or prestressed concrete structural members because of a few limitations; for example, the inelastic characteristics and the small relative velocity change over stress change in a concrete medium [6]. The theoretical study of elastic waves in an infinite prestressed solid that is homogeneous and isotropic is well-advanced $[7,8]$, but the deduced analytical equations offer no practically effective applications for nonhomogeneous and inelastic materials such as concrete under an inconsistent stress field. Thus, it is necessary to experimentally investigate and analyze the distribution of ultrasonic velocities in prestressed concrete structures in the field.

Generally, ultrasonic testing methods assess material conditions and deterioration by comparing velocity, attenuation, frequency, and energy against reference values such as ultrasonic pulse velocity (UPV) determined using laboratory specimens. The ratio of field UPV to the reference UPV 
indicates the level of material condition [9]. Traditionally, the pressure wave (P-wave) pulse velocity has been popularly applied to concrete structures for its easy generation and measurement. However, the statistical stability of experimental data for P-wave velocity is weaker than for shear (S-) or Rayleigh (R-) waves because the energy of a $\mathrm{P}$-wave is much less than that of S- and R-waves [10]. Furthermore, the Pwave depends on the presence of pore water in the concrete. Therefore, S- and R-wave pulse velocities have recently been applied to various structures.

For this study, we measured and analyzed P-, S-, and Rwave velocities in both reinforced and prestressed concrete slabs to investigate the statistical distribution of each wave velocity with and without prestressing. The experimental results present practical guidelines for applying the ultrasonic methods to reinforced and prestressed concrete structures.

\section{Ultrasonic Pulse Velocity and Its Measurement}

In an elastic and stress-free solid medium, body waves travel within the body (P- and S-waves), and surface waves (Rwaves) travel along the free surface of the material. The velocities of wave propagation depend on elastic constants and the mass density of the material. The relations among P-, $\mathrm{S}-$, and R-wave velocities can be expressed by the following equations [15]:

$$
\begin{aligned}
V_{\mathrm{P}} & =\sqrt{\frac{E(1-v)}{\rho(1+v)(1-2 v)}}, \\
V_{\mathrm{S}} & =\sqrt{\frac{E(1-v)}{2 \rho(1+v)}}, \\
V_{\mathrm{R}} & =\frac{0.87+1.12 v}{1+v} \sqrt{\frac{E}{\rho} \frac{1}{2(1+v)}},
\end{aligned}
$$

where $V_{\mathrm{P}}, V_{\mathrm{S}}$, and $V_{\mathrm{R}}$ are the velocities of P-, S-, and R-waves, respectively, and $E, \rho$, and $v$ are dynamic elasticity, density, and Poisson's ratio, respectively.

$V_{\mathrm{P}}$ typically ranges from $3500 \mathrm{~m} / \mathrm{s}$ to $4500 \mathrm{~m} / \mathrm{s}$ and $V_{\mathrm{R}}$ from 1800 to $2500 \mathrm{~m} / \mathrm{s}$ for sound concrete [16]. The motion resulting from $\mathrm{R}$-waves is restricted to a region near the surface and decreases exponentially in amplitude away from the surface. The R-wave penetration depth is inversely related to the frequency of the wave. R-waves are easily generated in a solid by a transient point source and more readily sensed than $\mathrm{P}$ - and S-waves because of their large amplitude. The only limitation of R-waves is caused by the limited penetration depth; the disturbances are confined to the surface region of thickness, approximately twice the wavelength, $\lambda_{R}$. Figure 1 shows the wave pressure field generated by a harmonic normal load applied at the interface. The propagating P-, S-, and R-waves in the concrete have 7, 26, and $67 \%$ of the source energy, respectively [11].

2.1. P-Wave (Pressure Wave) Measurement. The P-wave ultrasonic measurement mainly uses the arrival time of a stress wave and has two types of methods: direct (pulse-echo and through-transmission) and indirect transmission, as illustrated in Figure 2 [12]. The direct transmission uses the transfer of P-wave energy passing through a cross section between the transmitter and receiver. In the pulse-echo method, the transmitter/receiver transducer performs both sending and receiving of the pulsed waves reflected from the back wall of specimen. In the through-transmission, a transmitter sends ultrasound waves through one surface, and a receiver detects them on the opposite surface. On the other hand, the indirect transmission uses the P-wave path near surface. It is mainly used when only one face of the concrete is accessible or when the depth of a surface crack is to be determined. It is the least sensitive of the transducer arrangement for a given path. The surface response caused by the arrival of reflected or transmitted waves is monitored by the same or a different transducer acting as a receiver. The receiver output is displayed on an oscilloscope as a time-domain waveform. The round-trip travel time of the pulse can be calculated by determining the time from the transmitted pulse to the reflected echo. If the wave speed in the material is known, this travel time can be used to compute the depth of the reflecting interface. Transducers associated with short-duration, low-frequency $(50-200 \mathrm{kHz})$ focused waves are generally used for testing concrete.

2.2. S-Wave (Shear Wave) Measurement. One of the most effective ways to measure S-wave velocity is with MIRA equipment that generates shear wave tomography [13]. The MIRA also operates on the basic ultrasonic pulse-echo principle. Unlike other pulse-echo methods, the MIRA device does not require a coupling agent with a spring-loaded design. Interactive postprocessing software uses the synthetic aperture focusing technique (SAFT) to generate $2 \mathrm{D}$ and $3 \mathrm{D}$ images of the test results [13] (Figure 3). The generated Swaves have particle motion that propagates perpendicular to the direction of the wave front with a velocity about 60 percent that of P-waves [11]. Samokrutov et al. [17] showed that using $\mathrm{S}$-waves instead of $\mathrm{P}$-waves reduces the amount of backscattering and signal attenuation in the direction parallel to the propagating wave.

Recent advances have improved the MIRA transducer, which can now create a 3D tomography of internal defects that might be present in a concrete element, with penetration depths up to about $2.0 \mathrm{~m}$ [13]. MIRA is based on the ultrasonic pitch-catch method and uses an antenna of dry point contact transducers that emit shear waves into the concrete with a nominal center frequency of $50 \mathrm{kHz}$. Using an array of point transducers rapidly produces 180 transit time measurements during each test. The antenna is composed of a 4 by 12 array of point transducers and a control unit that operates the transducers, as illustrated in Figure 4 [14]. The transducers act as transmitters and receivers in a sequential mode. The use of very high frequencies with the pulse-echo method could be beneficial in terms of improved defect resolution.

2.3. R-Wave (Rayleigh Wave) Measurement. The conventional measurement of R-wave velocity uses the time difference 


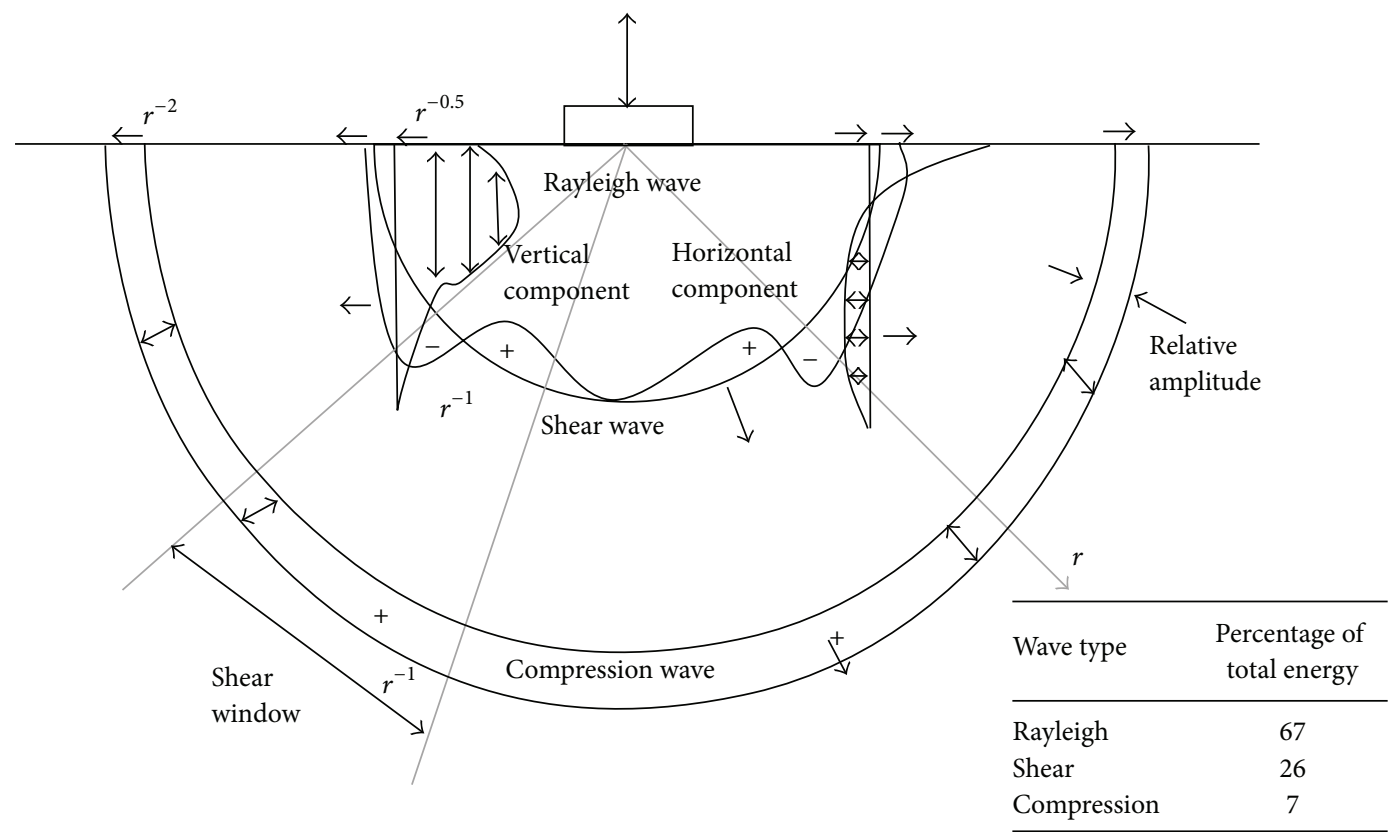

FIGURE 1: Distribution of displacement and energy in P-, S-, and R-waves from a harmonic normal load on a half-space for $v=0.25$ [11].

(a) Direct transmission
(1) Pulse-echo

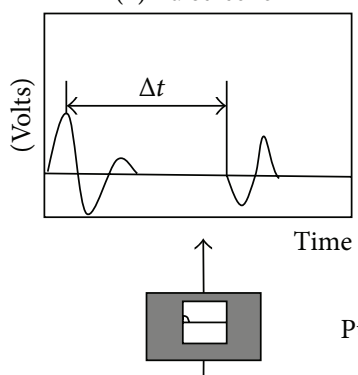

Transmitter/receiver
(2) Through-transmission

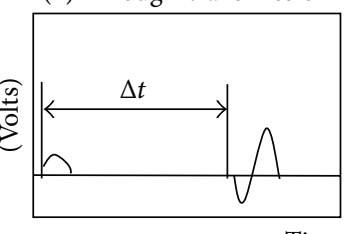

Time (b) Indirect transmission

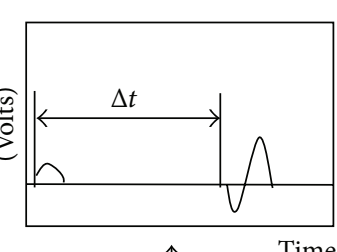

Time

Pulser/oscilloscope

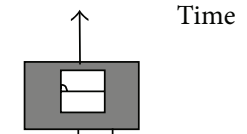

Transmitter

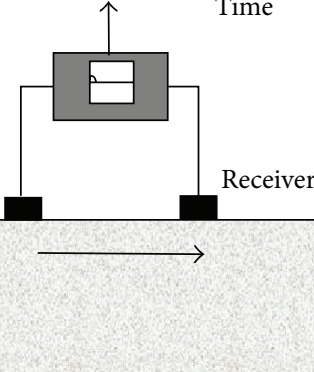

Receiver

FIGURE 2: Schematic of ultrasonic direct and indirect transmission method [12].

between the first or second significant peaks in two Rwaves from two receivers $[18,19]$. However, system errors in catching the peak points can occur. The best way to make up for that shortcoming uses the dispersion curve of Lamb waves in the plate with MASW (multichannel analysis of surface waves) [20, 21].

MASW data collection is based on $\mathrm{N}$-wave signals collected on the surface along a linear array of sensed points equally spaced (with spacing $d x$ ) from the wave source, such as the impact event illustrated in Figure 5. The impact event for wave generation must be provided by a high frequency impactor (small contact area). Multiple data are processed as individual signals obtained by each sensor. Data are transformed from the offset time-domain to the frequency-phase velocity domain using a 2D Fourier transform, producing a phase velocity that is usually called the dispersion curve.

MASW has been commonly applied to the surface wave testing of pavements, and the experimentally computed dispersion curve is interpreted to represent R-waves. Several modes of guided dispersive waves propagating in a pavement structure can be measured at the surface and used for material characterization, including the A0 and SO Lamb modes used to determine Poisson's ratio, R-wave velocity, and plate thickness [22]. An example of a dispersion curve in 


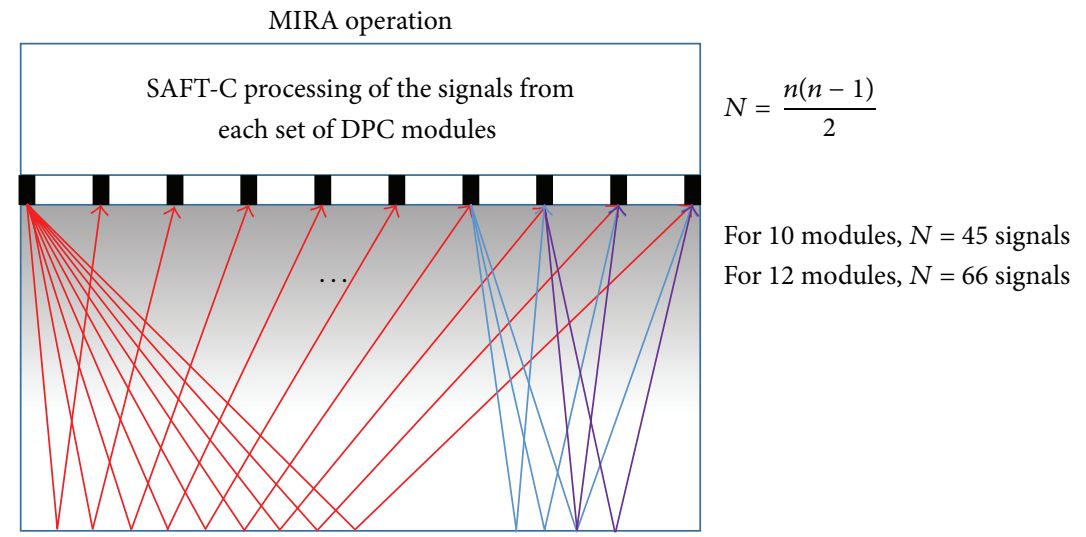

FIGURE 3: Schematic representation of the digitally focused array signal capturing scheme [13].

TABLE 1: Information on the prestress strand.

\begin{tabular}{lccccc}
\hline Tendon type & Diameter $(\mathrm{mm})$ & Area $\left(\mathrm{mm}^{2}\right)$ & Elongation $(\%)$ & Tensile strength $(\mathrm{MPa})$ & Jacking force $(\mathrm{kN})$ \\
\hline SWPC7B & 12.7 & 98.7 & 3.5 & 1860 & 128.5 \\
\hline
\end{tabular}

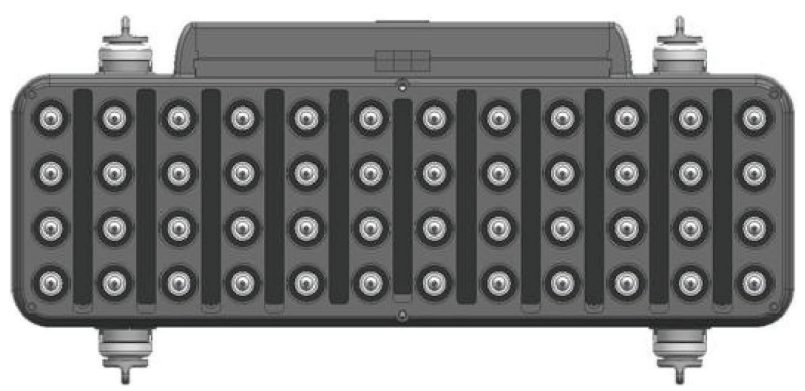

FIGURE 4: Illustration of low-frequency ultrasonic tomography (MIRA) [14].

a concrete slab with $30 \mathrm{~cm}$ depth is presented in Figure 6. It is obtained by using MASW wave transformation in the following equation [20]:

$$
S\left(\omega, c_{\mathrm{P}}\right)=\int e^{-i\left(\omega / c_{\mathrm{P}}\right) x} U(x, \omega) d x,
$$

where $U(x, \omega)$ is the normalized complex spectrum obtained from the Fourier transformation of $u(x, t)$, which is multichannel record at different offsets. $\omega$ is the angular frequency, and $c_{\mathrm{P}}$ is the phase velocity.

\section{Experiments}

3.1. Test Specimens. To simulate the effects of prestressing on UPV into a concrete slab, we carried out a series of ultrasonic tests on the three types of concrete slab (S1, S2, and S3) shown in Figure 7. All of the specimens are $3000 \mathrm{~mm} \times 3000 \mathrm{~mm}$ with a nominal thickness of $250 \mathrm{~mm}$, and they all contain transverse and longitudinal $13 \mathrm{~mm}$ diameter reinforcements with $560 \mathrm{~mm}$ spacing at 20 and $230 \mathrm{~mm}$ depths, respectively. We prestressed the S2 and S3 slabs in the two and one ways, respectively. Five $12.7 \mathrm{~mm}$ diameter strands with $350 \mathrm{~mm}$ spacing were applied in each direction. The details of the prestress strand are summarized in Table 1.

The specified w/c of the concrete mixture was 0.48 with $6 \%$ entrained air content. The mixture contained Type I cement and limestone aggregates with a maximum coarse aggregate size of $25 \mathrm{~mm}$. At the time of concrete placement, slump was measured as $150 \mathrm{~mm}$ and density as $2250 \mathrm{~kg} / \mathrm{m}^{3}$. The specimens were moist-cured for 7 days by covering them with saturated burlap with a bleed hose and plastic sheeting. Beyond the eighth day, the specimens were kept in laboratory air.

We determined the concrete's mechanical properties using tests performed on ten $150 \mathrm{~mm} \times 300 \mathrm{~mm}$ standard cylinders prepared from the same batch of concrete. For the same conditions as the S1, S2, and S3 slabs, we air-cured the standard specimens for 28-day strength, static modulus, and Poisson's ratio. We determined the compressive strength using ASTM C-39 [23] and conducted the modulus of elasticity $\left(E_{c}\right)$ and Poisson's ratio tests $(v)$ from ASTM C-469 [24]. The average results for the 28 -day tests were $14.84 \mathrm{MPa}$ for strength, $17.68 \mathrm{MPa}$ for the modulus of elasticity, and 0.16 for Poisson's ratio. Also, the dynamic modulus $\left(E_{d}\right)$ was $21.30 \mathrm{GPa}$, found by following the empirical relationship proposed by Lydon and Balendran [25]:

$$
E_{c}=0.83 E_{d}(\mathrm{GPa})
$$

\subsection{Test Methods}

3.2.1. P-Wave Measurement. We measured the $\mathrm{P}$-wave velocity of concrete, $V_{\mathrm{P}}$, according to BS 1881 [26] using a pair of P-wave transducers (MK-954 transmitters and receivers) connected to a pulse-receiver (Ultracon-170). The transmitter was driven by a $200 \mathrm{~V}$ square pulse, generating a transverse 


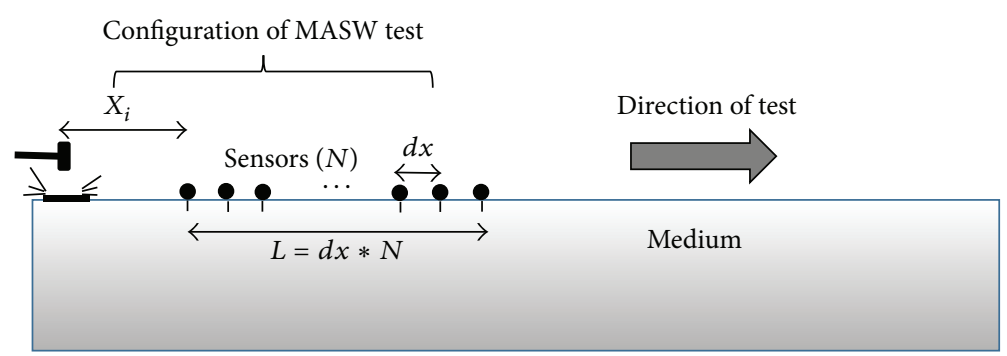

Figure 5: A scheme for an MASW test, in which $X_{i}$ is the near offset, $d x$ is the sensor spacing, and $L$ is the offset range.

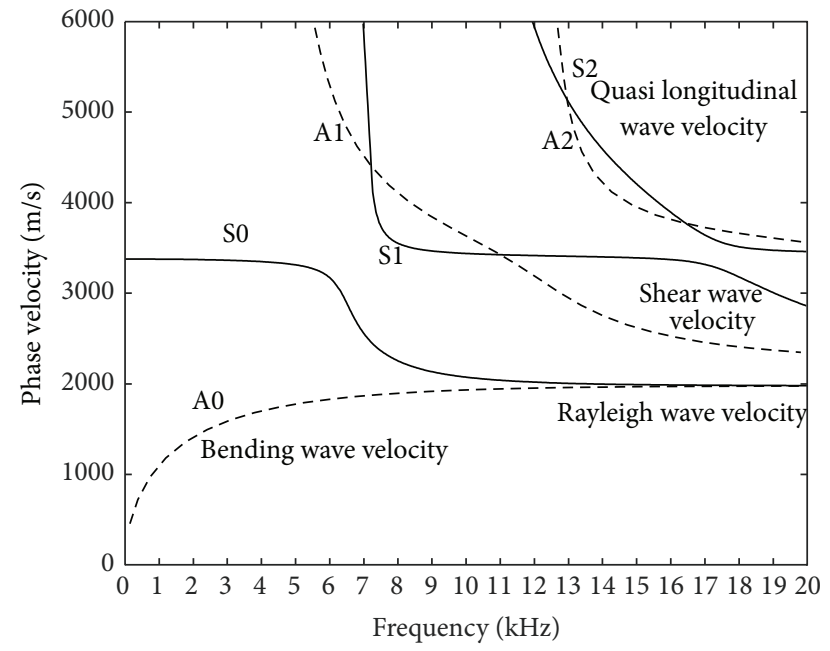

Figure 6: Phase velocity dispersion curves determined by MASW (based on the FE simulation of a concrete slab with a thickness of $0.3 \mathrm{~m}$ ). A density of $2250 \mathrm{~kg} / \mathrm{m}^{3}$, an elasticity of $23 \mathrm{GPa}$, and a fixed Poisson's ratio of 0.167 are assumed for the concrete slab.

ultrasonic pulse of $52 \mathrm{kHz}$. The receiver measured the transient stress waves through the surface of each slab (Figure 8).

We performed the P-wave measurements with a digital scope board synchronized with the pulser-receiver unit; therefore, data collection started at the time of pulse application. Measurement of flight time was affected by the electrical noise superimposed with the waveform. Therefore, to minimize random errors associated with identifying the arrival time, we performed wave averaging of 128 waveforms. The first step in identifying the flight time from the waveform was establishing the baseline. Afterward, the waveform was further smoothed using a 10-point moving average filter.

We made a total of 40 indirect measurements on each concrete slab using a coordinate system drawn on the slab surface (Figure 9). The coordinate system consisted of a primary grid at $353 \times 100 \mathrm{~mm}$ spacing. The gridlines were labeled along the width of the specimens as axes A, B, C, D, E, F, $\mathrm{G}$, and $\mathrm{H}$ and along the length of the specimens as axes I, II, III, IV, and V. We made indirect measurements longitudinally along the lettered axes. We placed the transmitter and receiver transducers at the grid nodes and measured the average P-wave velocity between them. For example, at A-I test position, upper and bottom circles represent the transducers (transmitter and receiver), and cross marks indicate the average P-wave velocity between two transducers.

We acquired the transmitter and conditioned receiver signals using a high-speed ( $1 \mathrm{MHz}$ sampling rate) analog to digital data acquisition board. We developed a computer algorithm based on a fixed threshold level to determine the time of flight using the digitally acquired waveforms. Figure 10 presents a typical signal of the transmitter and receiver transducers.

3.2.2. MIRA Test for the S-Wave Measurement. For effective $\mathrm{S}$-wave measurement, we used MIRA equipment that was verified in both Europe and the United States. The procedure is simple: a trigger button on the MIRA board starts reception of all possible combinations of pulse reflections from each test point location through the appropriate filtering and spacetime processing based on the SAFT algorithm. Eventually, the digital display presents a B-scan image with the S-wave velocity.

We made a total of 20 direct S-wave measurements on each concrete slab using the same coordinate system as for the P-wave measurement. However, we conducted these tests only in the areas shaded in Figure 11. We labeled the gridlines along the width of the specimens as axes $\mathrm{AB}, \mathrm{CD}, \mathrm{EF}$, and $\mathrm{GH}$ and those along the length of the specimens as axes I, II, III, IV, and V. We carried out direct measurements at a single position because the MIRA uses the pulse-echo method (Figure 12).

Figure 13 shows the result of the MIRA testing at the EF-V point on the S1 slab. The reconstructed B-scan image depicts an average transverse cross section $\left(90^{\circ}\right.$ from the transducer array travel path). In the view, the center of the bright red color represents reflections from the back of the slab, which corresponds to a nominal thickness of $250 \mathrm{~mm}$. Additional reverberations from the bottom of the slab, at approximately twice the thickness of each consecutive step, are observed at the $500 \mathrm{~mm}$ depth. Also, the average S-wave velocity computed by the SAFT algorithm is displayed in the left corner.

3.2.3. MASW Test for R-Wave Measurement. We applied an MASW testing configuration for the identification of R-wave velocity consistently to the three test specimens along the 12 paths shown in Figure 14.

We used a steel ball with an $18 \mathrm{~mm}$ diameter as the impact source for each MASW test. The forcing function 

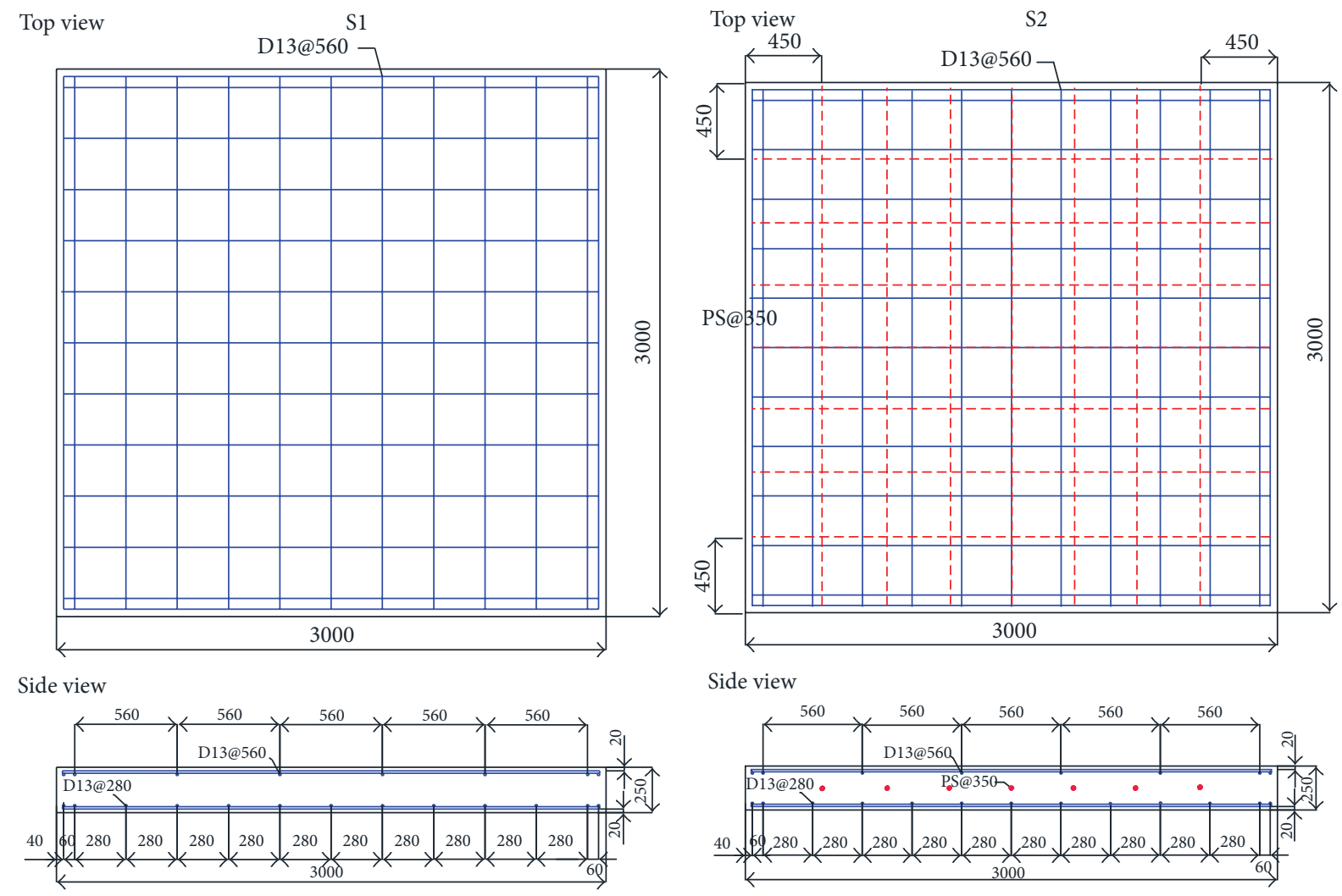

Side view
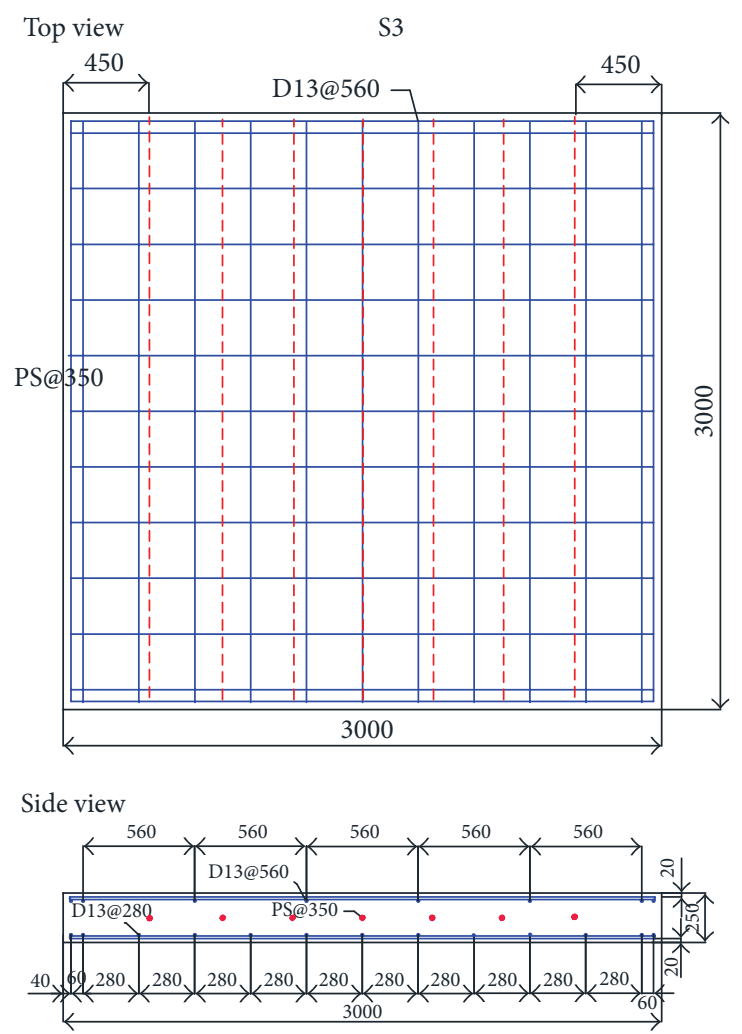

FIGURE 7: The plan and side views of specimens S1, S2, and S3. All dimensions in millimeters. 


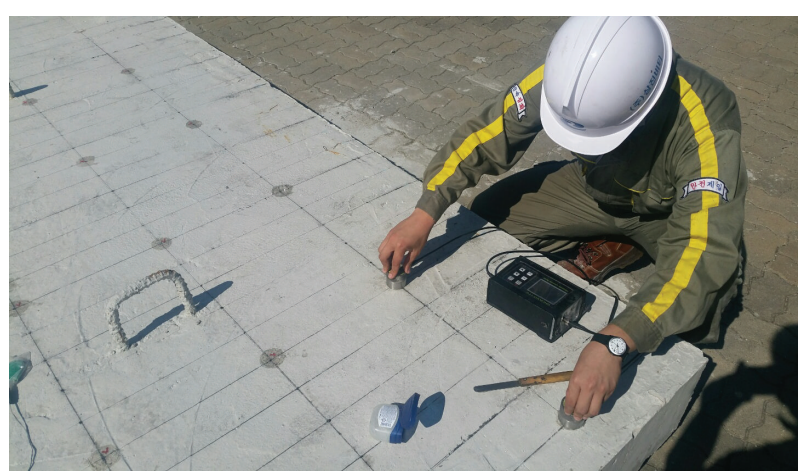

Figure 8: P-wave measurement in a grid on the concrete slab surface.

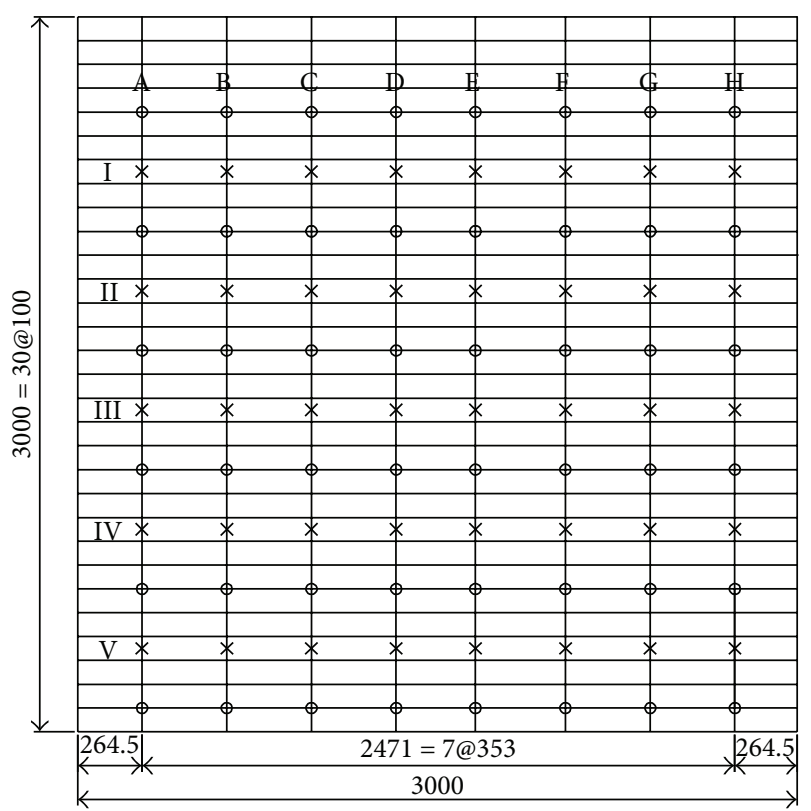

FIGURE 9: A plan view of the $353 \times 100 \mathrm{~mm}$ test point grid for the $\mathrm{P}$-wave measurement. The test was carried out on the grid locations. The $\circ$ (circle) and $\times$ (cross mark) indicate the positions of the transducers and average measurements, respectively.

associated with the impact exhibits consistent and broad spectral content, ranging from DC to $15 \mathrm{kHz}$. We used 5 accelerometers (PCB 353B16), with a $\pm 5 \%$ frequency range of 1 to $10 \mathrm{kHz}$ and a resonance frequency around $70 \mathrm{kHz}$, in even $10 \mathrm{~cm}$ spacing along the test line to detect the surface vibrations generated by the ball impact event. For a rapid test, we mounted the 5 accelerometers to the frame, as shown in Figure 15. We stabilized the signals using a signal conditioner (PCB 482C16) and digitized them at a sampling frequency of $1 \mathrm{MHz}$ using an NI-PXI 5105 oscilloscope.

The multichannel record consists of 5 time series (called traces) from the receivers in an ordered manner. MASW data processing consists of three steps: (1) detection of surface waves, (2) construction of the dispersion image and extraction of the signal dispersion curve, and (3) identification of the several Lamb wave modes and R-wave velocity. All these

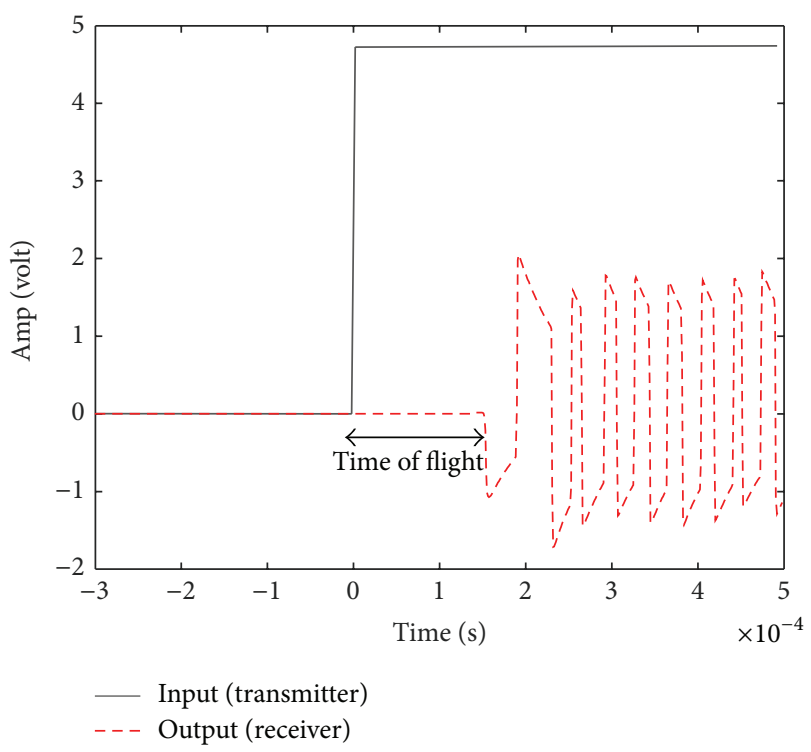

FIgure 10: Procedure for determining P-wave time of flight at the A-II point in slab S1.

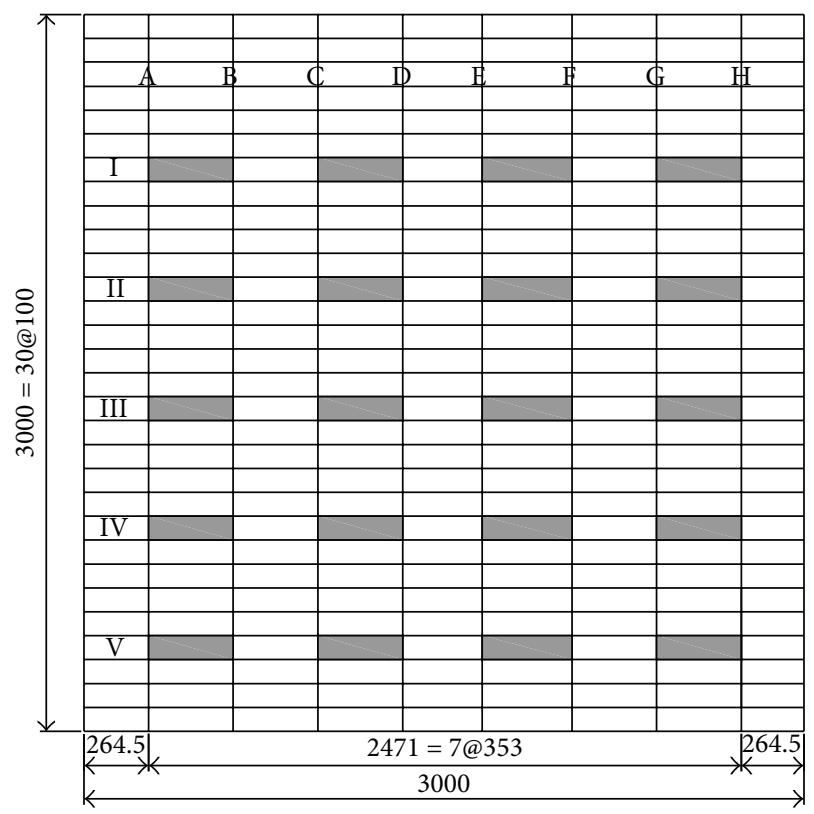

Figure 11: A plan view of the $353 \times 100 \mathrm{~mm}$ test point grid for the $\mathrm{S}$-wave measurement. The test was carried out at the shaded areas in the grid system.

steps can be fully automated. The detection of surface waves examines the recorded seismic waves in the most probable range of frequencies and phase velocities. Construction of the phase velocity image is accomplished through a $2 \mathrm{D}$ (time and pace) wave field transformation method. This transformation eliminates all the ambient noise from human activities as well as source-generated noise such as scattered waves. The necessary dispersion curve, such as that of the $\mathrm{A} 0, \mathrm{Al}, \mathrm{S} 0$, or $\mathrm{S} 1$ fundamental-mode, is then extracted from the accumulation pattern. The extracted dispersion curve is 


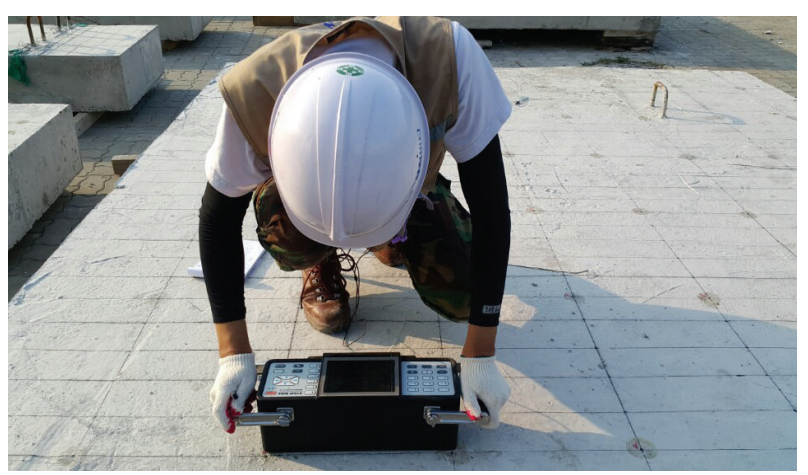

FIGURE 12: S-wave measurement in the grid on concrete slab surface.

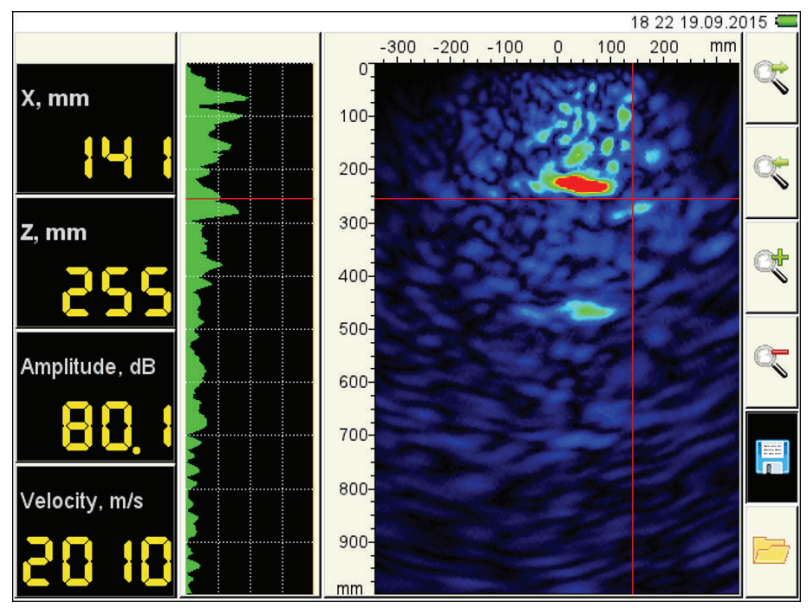

Figure 13: B-scan image from MIRA at the EF-V point in slab S1.

finally used as a reference to identify the R-wave velocity. For each test, we obtained time signals of $10 \mathrm{~ms}$ duration. Because the amplitude of the input forcing function at each test point across the concrete surface is inherently inconsistent, we normalized the amplitude of each time signal with respect to the early-arriving negative peak of the R-wave pulse arrival within that signal, which provides more consistent MASW data.

Figure 16 shows the experimental results along path 6 in the S2 slab and exhibits dispersion images and curves up to $12 \mathrm{kHz}$. The Lamb wave curves in the single layer must be fit to one of the responses. The A0 and S0 modes beyond $10 \mathrm{kHz}$ converge to about $2000 \mathrm{~m} / \mathrm{s}$ phase velocity, corresponding to the R-wave. Also, the impact-echo mode (thickness mode), which is identical to the $\mathrm{S} 1$ mode, shows that $7.5 \mathrm{kHz}$ corresponds to a nominal thickness of $250 \mathrm{~mm}$.

\section{Results and Discussion}

4.1. The Experimental Data for P-, S-, and R-Wave Velocities. To minimize the random errors associated with identifying the arrival time, we averaged the times for 128 waveforms for the P-wave measurement. For the S-wave measurement, we repeated the MIRA tests at each point until we identified a clear reflection from the back of the slab. We also carried

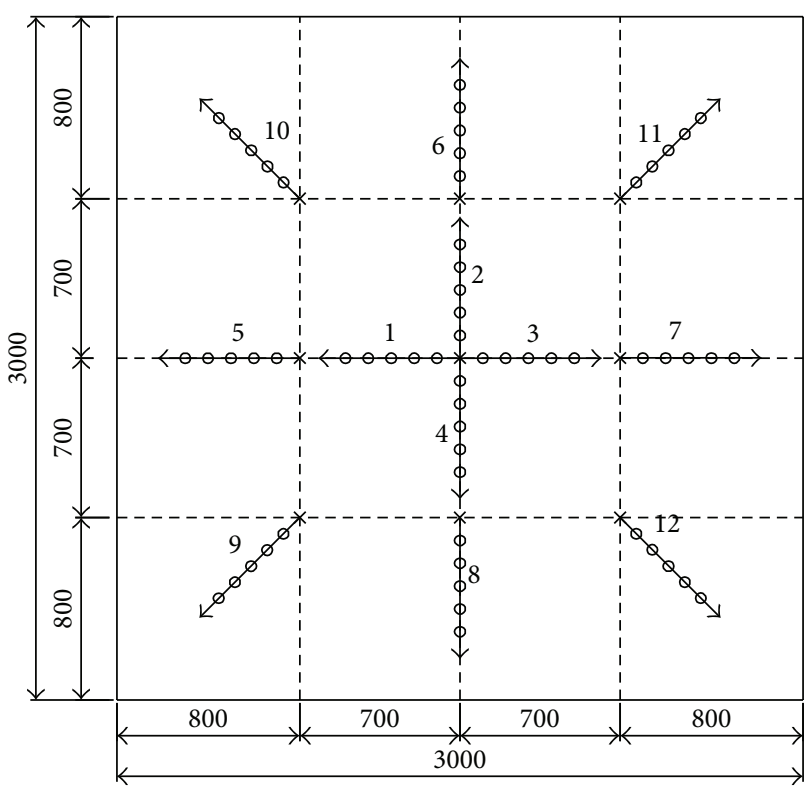

FIGURE 14: A plan view of the 12 test paths for the R-wave measurement. The $\circ$ marks indicate the sensor positions in each path, and the arrows show the direction of each test.

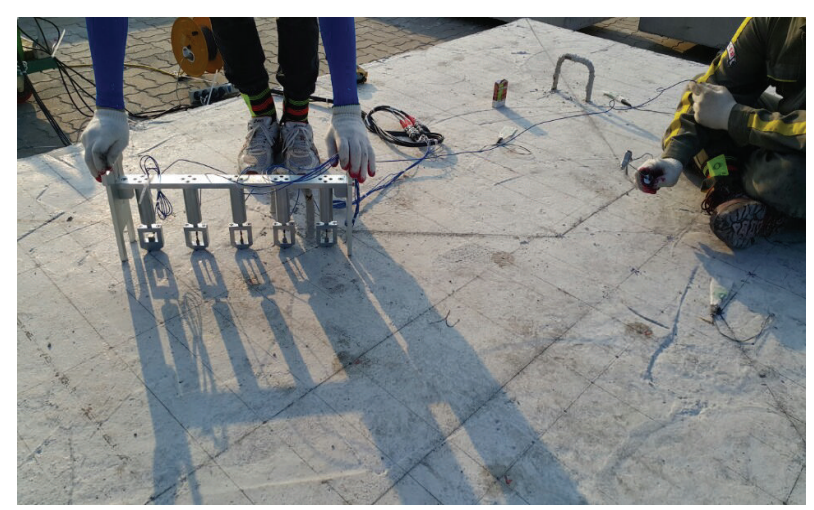

Figure 15: MASW test for the R-wave measurement along predefined paths on the concrete slab surface.

out the MASW test on a single path until the computed dispersion image showed a distinct A0 mode. All the data for the P-, S-, and R-wave velocities are summarized in Tables 24.

The ranges for $\mathrm{P}-$, S-, and R-wave velocities are $3002-$ $3472 \mathrm{~m} / \mathrm{s}, 1780-2160 \mathrm{~m} / \mathrm{s}$, and $1753-1988 \mathrm{~m} / \mathrm{s}$, respectively, for the reinforced concrete slab (S1). The data distribution for each type of wave is reasonably comparable to the theoretical velocity values of 3174,1851 , and $1827 \mathrm{~m} / \mathrm{s}$ computed by (1) with Poisson's ratio (0.16), dynamic elasticity (21.3 MPa), and density $\left(2250 \mathrm{~kg} / \mathrm{m}^{3}\right)$. The difference between the maximum and minimum velocities in slab S1 tends to be smaller than those in S2 and S3, which have prestressing in two and one ways, respectively, because the stress field in the reinforced concrete slab is more consistent than those in the prestressed slabs. 
TABLE 2: P-wave velocities (m/s) at test grid points in slabs $\mathrm{S} 1, \mathrm{~S} 2$, and S3.

\begin{tabular}{|c|c|c|c|c|c|c|c|c|c|c|c|c|c|c|c|}
\hline \multirow{2}{*}{ Test point } & \multicolumn{5}{|c|}{$\mathrm{S} 1$} & \multicolumn{5}{|c|}{ S2 } & \multicolumn{5}{|c|}{ S3 } \\
\hline & I & II & III & IV & $\mathrm{V}$ & I & II & III & IV & $\mathrm{V}$ & I & II & III & IV & V \\
\hline A & 3002 & 3301 & 3295 & 3326 & 3231 & 3411 & 3258 & 3400 & 3183 & 3391 & 3253 & 3320 & 3348 & 3358 & 3244 \\
\hline B & 3136 & 3333 & 3283 & 3331 & 3308 & 3333 & 3433 & 3395 & 3285 & 3691 & 3211 & 3733 & 3630 & 3300 & 3401 \\
\hline $\mathrm{C}$ & 3254 & 3197 & 3281 & 3350 & 3322 & 3275 & 3335 & 3287 & 3287 & 3358 & 3316 & 3409 & 3367 & 3256 & 3260 \\
\hline $\mathrm{D}$ & 3429 & 3339 & 3318 & 3340 & 3429 & 3329 & 3333 & 3297 & 3348 & 3246 & 3301 & 3256 & 3283 & 3301 & 3337 \\
\hline $\mathrm{E}$ & 3130 & 3268 & 3104 & 3472 & 3378 & 3166 & 3398 & 3300 & 3474 & 3160 & 3554 & 3337 & 3435 & 3341 & 3406 \\
\hline $\mathrm{F}$ & 3108 & 3268 & 3300 & 3391 & 3374 & 3374 & 3528 & 3280 & 3524 & 3260 & 3320 & 3458 & 3130 & 3279 & 3371 \\
\hline G & 3191 & 3207 & 3207 & 3234 & 3275 & 3277 & 3312 & 3295 & 3540 & 3343 & 3358 & 3329 & 3367 & 3379 & 3371 \\
\hline $\mathrm{H}$ & 3334 & 3374 & 3271 & 3333 & 3363 & 3255 & 3400 & 3793 & 3818 & 3316 & 3316 & 3497 & 3444 & 3442 & 3447 \\
\hline Min & & & 3002 & & & & & 3160 & & & & & 3130 & & \\
\hline Max & & & 3472 & & & & & 3818 & & & & & 3733 & & \\
\hline $\operatorname{Avg}(\mathrm{m} / \mathrm{s})$ & & & 3285 & & & & & 3367 & & & & & 3362 & & \\
\hline Std $(\mathrm{m} / \mathrm{s})$ & & & 97 & & & & & 146 & & & & & 110 & & \\
\hline
\end{tabular}

Avg = average velocity and Std = standard deviation.

TABLE 3: S-wave velocities $(\mathrm{m} / \mathrm{s})$ at test grid points in slabs $\mathrm{S} 1, \mathrm{~S} 2$, and S3.

\begin{tabular}{|c|c|c|c|c|c|c|c|c|c|c|c|c|}
\hline \multirow{2}{*}{ Test point } & \multicolumn{4}{|c|}{ S1 } & \multicolumn{4}{|c|}{ S2 } & \multicolumn{4}{|c|}{ S3 } \\
\hline & $\mathrm{AB}$ & $\mathrm{CD}$ & $\mathrm{EF}$ & $\mathrm{GH}$ & $\mathrm{AB}$ & $\mathrm{CD}$ & $\mathrm{EF}$ & $\mathrm{GH}$ & $\mathrm{AB}$ & $\mathrm{CD}$ & $\mathrm{EF}$ & $\mathrm{GH}$ \\
\hline $\mathrm{I}$ & 1830 & 1870 & 2020 & 1800 & 2270 & 1790 & 1830 & 2320 & 1910 & 1930 & 1890 & 1900 \\
\hline II & 1950 & 1870 & 1840 & 1780 & 1800 & 1790 & 2070 & 1800 & 1930 & 1810 & 1900 & 2030 \\
\hline III & 1880 & 1840 & 1840 & 1900 & 1780 & 1800 & 1830 & 2050 & 1960 & 2040 & 1950 & 2010 \\
\hline IV & 1890 & 2160 & 1890 & 1890 & 1840 & 2070 & 2150 & 1920 & 1890 & 1980 & 1970 & 2050 \\
\hline V & 2020 & 1890 & 2010 & 2040 & 2110 & 2040 & 2040 & 1920 & 1890 & 2010 & 2010 & 1970 \\
\hline Min & \multicolumn{4}{|c|}{1780} & \multicolumn{4}{|c|}{1780} & \multicolumn{4}{|c|}{1810} \\
\hline Max & \multicolumn{4}{|c|}{2160} & \multicolumn{4}{|c|}{2320} & \multicolumn{4}{|c|}{2050} \\
\hline $\operatorname{Avg}(\mathrm{m} / \mathrm{s})$ & \multicolumn{4}{|c|}{1911} & \multicolumn{4}{|c|}{1961} & \multicolumn{4}{|c|}{1952} \\
\hline $\operatorname{Std}(\mathrm{m} / \mathrm{s})$ & \multicolumn{4}{|c|}{95} & \multicolumn{4}{|c|}{169} & \multicolumn{4}{|c|}{62} \\
\hline
\end{tabular}

Avg $=$ average velocity and Std $=$ standard deviation .

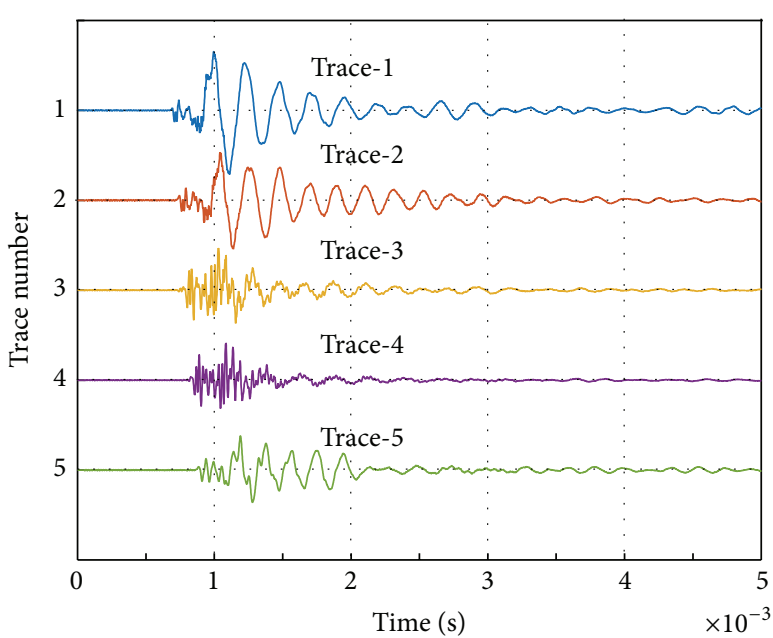

(a) The signals in the time domain

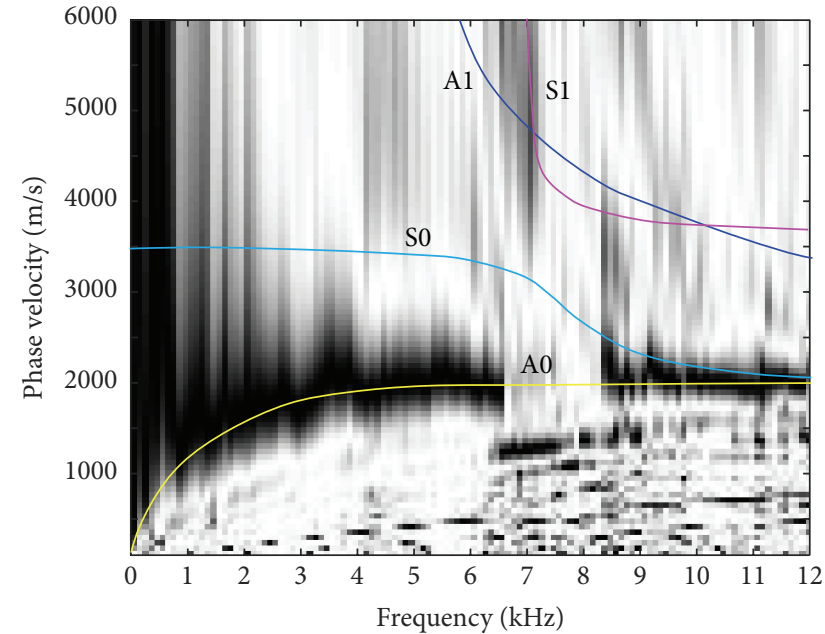

(b) Dispersion curve

FIGURE 16: MASW results along the 6th path in slab S2. The computed A0, A1, S0, and S1 Lamb modes corresponding to the slab depth overlap on the dispersion curve image. 
TABLE 4: R-wave velocities (m/s) along 12 paths in slabs S1, S2, and S3.

\begin{tabular}{|c|c|c|c|c|c|c|c|c|c|c|c|c|c|c|c|c|}
\hline \multirow{2}{*}{ Slab } & \multicolumn{12}{|c|}{ Path } & \multirow[b]{2}{*}{ Min } & \multirow[b]{2}{*}{ Max } & \multirow[b]{2}{*}{ Avg } & \multirow[b]{2}{*}{ Std } \\
\hline & 1 & 2 & 3 & 4 & 5 & 6 & 7 & 8 & 9 & 10 & 11 & 12 & & & & \\
\hline S1 & 1768 & 1855 & 1913 & 1753 & 1951 & 1835 & 1988 & 1894 & 1882 & 1921 & 1917 & 1882 & 1753 & 1988 & 1880 & 69 \\
\hline S2 & 2025 & 1951 & 1874 & 1988 & 2006 & 1992 & 1894 & 2043 & 1855 & 1795 & 1932 & 1855 & 1795 & 2043 & 1934 & 79 \\
\hline S3 & 1853 & 1988 & 1970 & 1917 & 1992 & 1894 & 1855 & 1971 & 1845 & 1932 & 2006 & 1888 & 1845 & 2006 & 1926 & 75 \\
\hline
\end{tabular}

Avg = average velocity and Std $=$ standard deviation.

TABLE 5: The results of statistical analysis for slabs S1, S2, and S3.

\begin{tabular}{|c|c|c|c|c|c|c|c|c|c|}
\hline \multirow{2}{*}{$\begin{array}{l}\text { Type of } \\
\text { measurement }\end{array}$} & \multirow[b]{2}{*}{ Type of slab } & \multicolumn{4}{|c|}{ Statistics } & \multicolumn{4}{|c|}{ Goodness-of-fit test } \\
\hline & & Sample size & $\begin{array}{c}\operatorname{Avg}(\sigma)^{*} \\
\mathrm{~m} / \mathrm{s}\end{array}$ & $\begin{array}{c}\operatorname{Std}(\mu)^{* *} \\
\mathrm{~m} / \mathrm{s}\end{array}$ & $\mathrm{COV}^{* * *}$ & $D_{\max }$ & $D_{5 \%}$ & $p$ value & Probability distribution \\
\hline \multirow{3}{*}{ P-wave } & S1 & 40 & 3285 & 97 & 0.030 & 0.16 & 0.21 & 0.20 & Normal \\
\hline & S2 & 40 & 3367 & 146 & 0.043 & 0.20 & 0.21 & 0.05 & Normal \\
\hline & S3 & 40 & 3362 & 110 & 0.033 & 0.19 & 0.21 & 0.09 & Normal \\
\hline \multirow{3}{*}{ S-wave } & S1 & 20 & 1911 & 95 & 0.049 & 0.23 & 0.29 & 0.18 & Normal \\
\hline & S2 & 20 & 1961 & 169 & 0.086 & 0.24 & 0.29 & 0.17 & Normal \\
\hline & S3 & 20 & 1952 & 62 & 0.032 & 0.20 & 0.29 & 0.33 & Normal \\
\hline \multirow{3}{*}{ R-wave } & S1 & 12 & 1880 & 69 & 0.037 & 0.18 & 0.37 & 0.76 & Normal \\
\hline & S2 & 12 & 1934 & 79 & 0.041 & 0.20 & 0.37 & 0.65 & Normal \\
\hline & S3 & 12 & 1918 & 75 & 0.039 & 0.22 & 0.37 & 0.51 & Normal \\
\hline
\end{tabular}

$\operatorname{Avg}(\sigma)^{*}=$ average velocity; $\operatorname{Std}(\mu)^{* *}=$ standard deviation; $\mathrm{COV}^{* * *}=$ coefficient of variable $(=\sigma / \mu \times 100(\%))$.

4.2. Statistical Analysis. We conducted our statistical analysis on the UPV data mathematically to evaluate variation in the experimental data from the reinforced and prestressed concrete slabs. We used the sample size, mean, and standard deviation of the UPV from slabs S1, S2, and S3 for the basic input, as summarized in Table 5.

Generally, for an ultrasonic wave at $50 \mathrm{kHz}$ with a wavelength of approximately $90 \mathrm{~mm}$, the time of flight between the transmitter and receiver transducers can be influenced by the nonuniformity of coarse aggregate distribution, which creates a basic data error in the concrete material [26]. The statistical data show that the standard deviations and COVs of R- and $\mathrm{S}$-waves are usually less than those of P-wave because of the two reasons. Firstly, P-wave has the highest velocity among all the ultrasonic waves. Secondly, the R- and S-waves depend less on boundary conditions and water pore pressure than Pwaves. Therefore, it is possible to measure more distinct flight time, resulting in the more accurate wave velocity. Moreover, the MASW and MIRA tests for R- and S-wave measurements use improved algorithms to minimize noise signals from the multiple-sensor measurements. Thus, P-wave measurement of a single wave path associated with a single sensor has more systematic and electrical errors than R- and S-wave measurements.

All the COVs of the UPV data in the field concrete specimen are good (4.0 to 5.0) or very good (3.0 to 4.0), according to ACI 214R [27], except for the S-wave measurement on slab S2. The large COV (0.086) in the S-wave measurement might be caused by unstable convergence in the SAFT algorithm on the rough surface of the concrete slab or by the inconsistent stress field in the prestressed concrete slab, even though we repeated the MIRA tests several times.

In all the UPV tests, the COV tends to grow larger as the prestressing becomes stronger because the stress field in prestressed slabs is inconsistent, and the analytical characterization of ultrasonic waves in the inconsistent stress field of a nonhomogeneous material is almost impossible. Thus, our purpose in this study was to experimentally identify the statistical variations in UPV data from prestressed slabs and suggest practical recommendations.

We also applied the Kolmogorov-Smirnov goodness-offit test [28] to all the cases to check the statistical distribution of the UPV measurements. In this test (as shown in Figures 17-19), we assumed an empirical stepwise cumulative frequency distribution function and plotted the cumulative normal distribution function for each of the variables presented in Table 5. The observed $D$-statistic, $D_{\max }$, was less than the critical value corresponding to the commonly used $5 \%$ level of significance (Table 5). Therefore, the null hypothesis that the data have a normal distribution cannot be rejected at the $5 \%$ level of significance. The $p$ value represents the probability that the observed sample statistic is equal to or more extreme than a statistical hypothesis, which means that the null hypothesis can be more accepted as the $p$ value becomes larger than the level of significance. For all the data, the null hypothesis was satisfied. Also, the experimental data for the R-wave are more statistically significant than those for the $\mathrm{S}$ - and P-waves. 

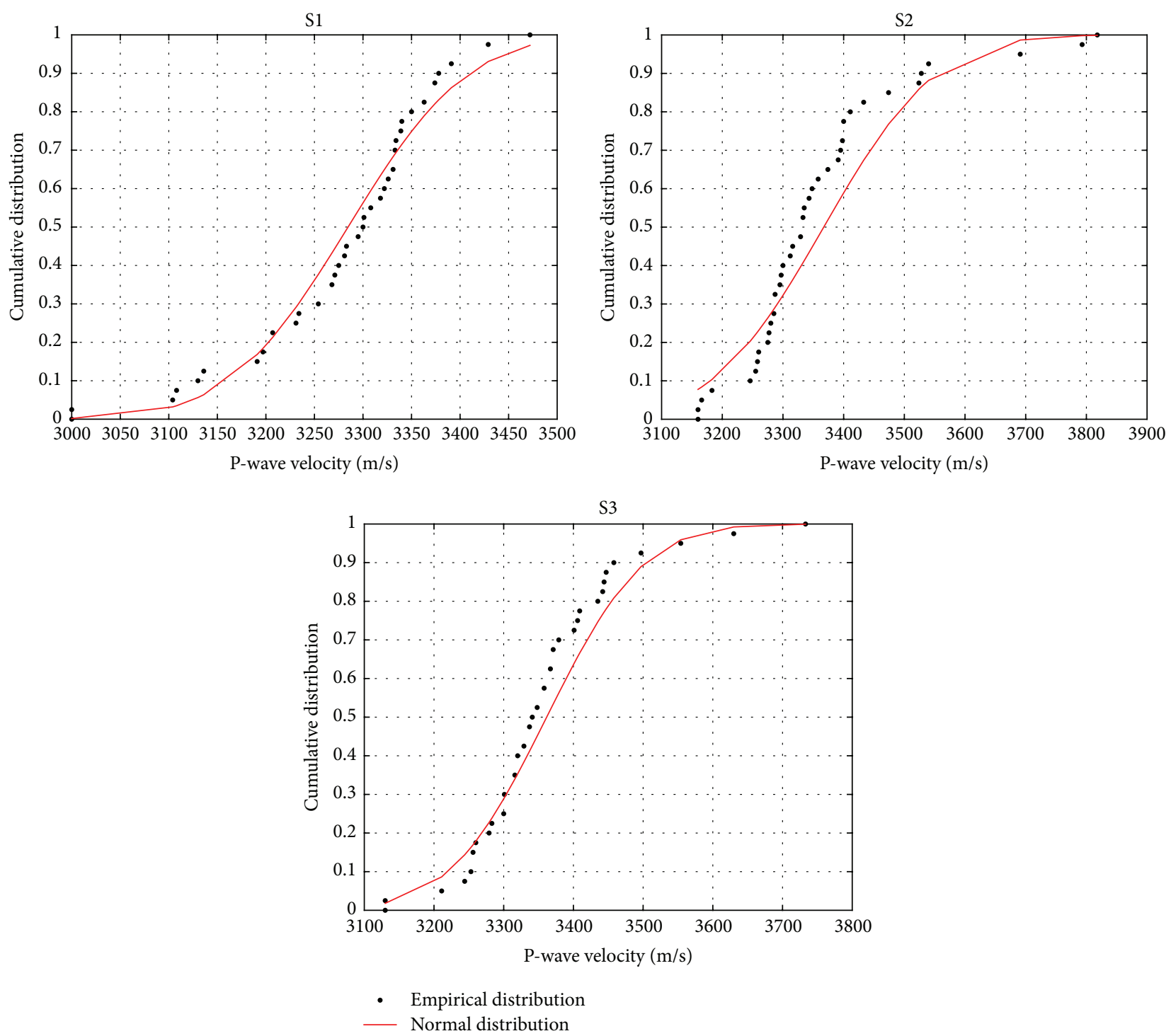

FIGURE 17: Cumulative normal distribution function of P-wave velocities.

To sum it all up, our inference is that all the types of UPV data can be represented as normally distributed variables with their respective means and standard deviations.

4.3. Comparison with the Dynamic Modulus from Static Test. We checked the accuracy of our experimental data by comparing the dynamic moduli from the measured UPV to one determined from the static modulus. Table 6 shows the comparison of the dynamic moduli for S1, S2, and S3. The error range for slab $\mathrm{S} 1$ (without prestressing) is $5-7 \%$, and the R-, S-, and P-waves, in that order, have smaller errors compared with the dynamic modulus from the static test. However, the error increases 12\% for S2 and S3 regardless of the wave type, even though error between S2 and S3 is below $2 \%$. Thus, prestressing affects all the types of wave velocities and increases the dynamic modulus by more than $10 \%$ based on the wave velocity.

\section{Conclusion}

We conducted a series of experimental UPV tests to explore the variation of $\mathrm{P}-, \mathrm{S}$-, and $\mathrm{R}$-wave velocities in a reinforced concrete slab and two prestressed concrete slabs. For the $\mathrm{S}$ - and R-wave measurements, we applied the MIRA and MASW tests, respectively, and verified that they are robust, promising test methods for wave velocity measurement. All of our experimental data are statistically stable and significant. Based on the data presented in this study, we draw the following conclusions.

(i) First, all the experimental UPV data are in the reasonable range theoretically computed from the physical properties found in the static test. The experimental data for the R-, S-, and P-waves, in that order, are statistically stable because of differences in energy magnitude, effects of confinement, and 
TABLE 6: Comparison of dynamic moduli from ultrasonic and static tests.

\begin{tabular}{|c|c|c|c|c|c|c|c|c|}
\hline \multirow[b]{2}{*}{ Measurement } & \multirow{2}{*}{ Slab } & \multicolumn{2}{|c|}{ Ultrasonic test } & \multicolumn{4}{|c|}{ Static test } & \multirow{2}{*}{ Error } \\
\hline & & $\begin{array}{l}\text { Avg } \\
\mathrm{m} / \mathrm{s}\end{array}$ & $\mathrm{Edu}^{*}(\mathrm{GPa})$ & $v$ & $\mathrm{fck}^{* *}(\mathrm{Mpa})$ & $E_{c}(\mathrm{GPa})$ & $E_{d}(\mathrm{GPa})$ & \\
\hline \multirow{3}{*}{$\mathrm{P}$-wave } & S1 & 3285 & 22.80 & 0.16 & 14.84 & 17.68 & 21.30 & $7.01 \%$ \\
\hline & S2 & 3367 & 23.96 & 0.16 & 14.84 & 17.68 & 21.30 & $12.46 \%$ \\
\hline & S3 & 3362 & 23.88 & 0.16 & 14.84 & 17.68 & 21.30 & $12.09 \%$ \\
\hline \multirow{3}{*}{ S-wave } & $\mathrm{S} 1$ & 1910 & 22.67 & 0.16 & 14.84 & 17.68 & 21.30 & $6.43 \%$ \\
\hline & S2 & 1960 & 23.87 & 0.16 & 14.84 & 17.68 & 21.30 & $12.07 \%$ \\
\hline & S3 & 1950 & 23.63 & 0.16 & 14.84 & 17.68 & 21.30 & $10.93 \%$ \\
\hline \multirow{3}{*}{ R-wave } & S1 & 1880 & 22.55 & 0.16 & 14.84 & 17.68 & 21.30 & $5.86 \%$ \\
\hline & S2 & 1934 & 23.87 & 0.16 & 14.84 & 17.68 & 21.30 & $12.06 \%$ \\
\hline & S3 & 1918 & 23.46 & 0.16 & 14.84 & 17.68 & 21.30 & $10.15 \%$ \\
\hline
\end{tabular}

$\mathrm{Edu}^{*}$ : dynamic modulus by ultrasonic test, $\mathrm{fck}^{* *}: 28$-day compressive strength.
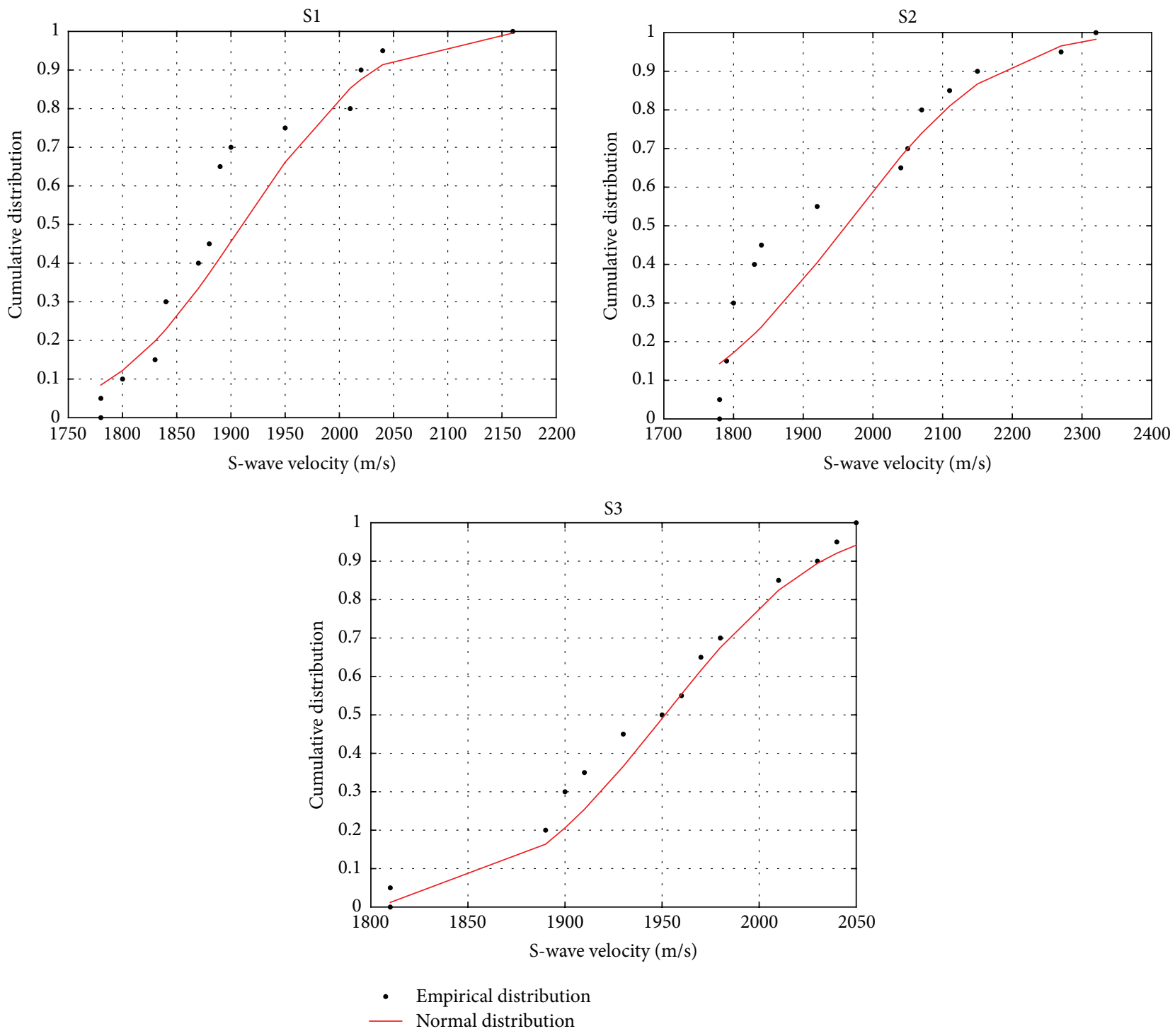

FIgURE 18: Cumulative normal distribution function of S-wave velocities. 

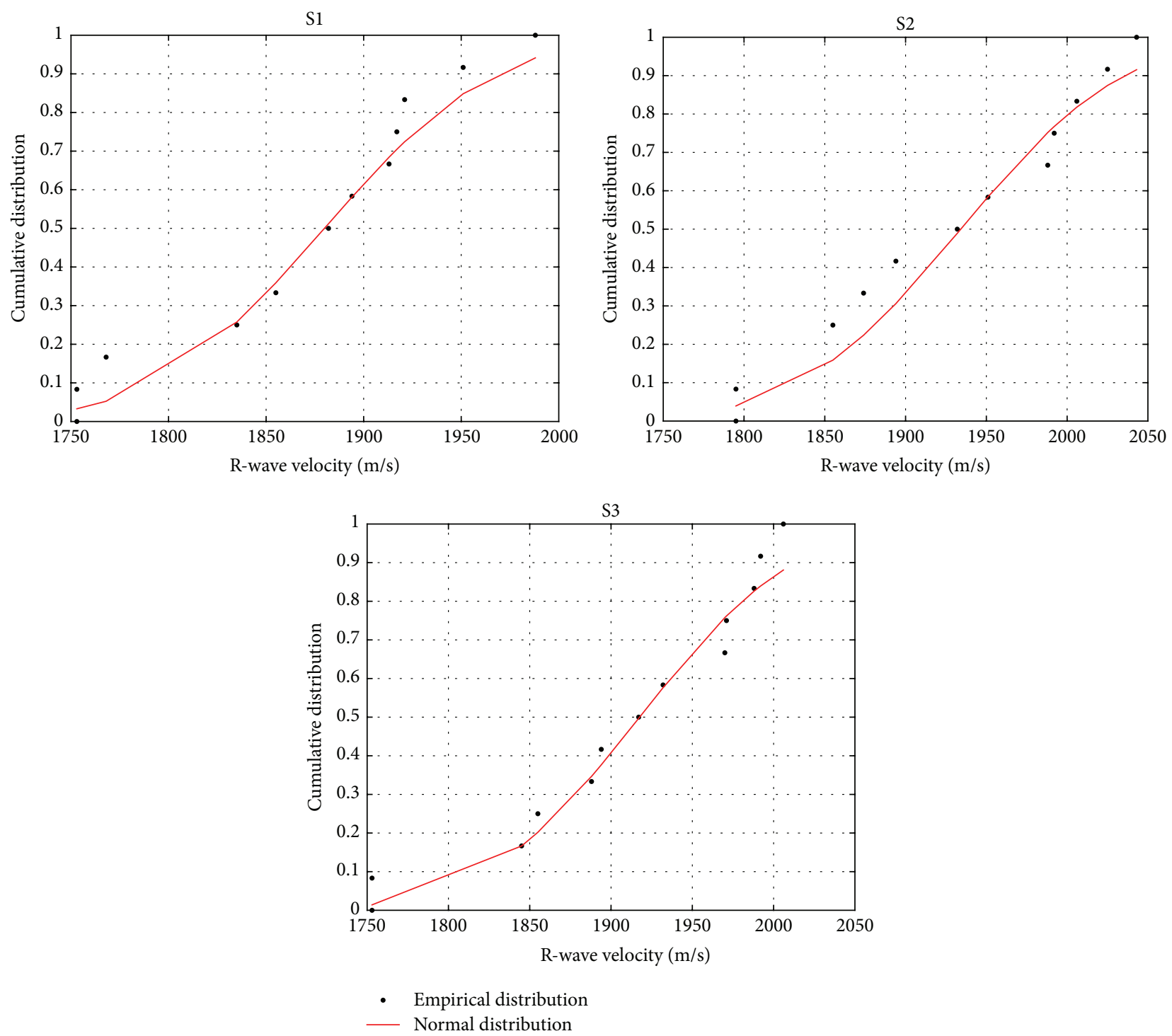

FIGURE 19: Cumulative normal distribution function of R-wave velocities.

improvements in the measurement equipment and analysis associated with the various sensors.

(ii) The statistical analysis by COV and goodness-of-fit test showed good or very good data distribution rates, according to ACI 214R, and all the data represent the normal distribution at the $5 \%$ level of significance. Thus, all of the data can be trusted, statistically.

(iii) The ultrasonic velocities in the prestressed concrete, regardless of wave type, are $2-3 \%$ faster than those in the reinforced concrete slab. Also, the dynamic moduli from the UPV data in the PSC slab are 5-7\% higher than those in the RC slab.

(iv) We obtained statistically reliable data using promising methods and showed the variation of elastic waves in PSC and RC slabs. Our experimental results offer practical guidelines for the application of ultrasonic methods to PSC structures.

\section{Competing Interests}

The authors declare that they have no competing interests.

\section{Acknowledgments}

This work was supported by a National Research Foundation of Korea (NRF) grant funded by the Korean Government (MSIP) (NRF-2013R1A2A2A01067754).

\section{References}

[1] J. S. Popovics, "A study of static and dynamic modulus of elasticity of concrete," ACI-CRC Final Report, 2008.

[2] I. O. Yaman, G. Inci, N. Yesiller, and H. M. Aktan, "Ultrasonic pulse velocity in concrete using direct and indirect transmission," ACI Materials Journal, vol. 98, no. 6, pp. 450-457, 2001.

[3] J. L. Rose, Ultrasonic Waves in Solid Media, Cambridge University Press, Cambridge, UK, 1999. 
[4] R. A. Toupin and B. Bernstein, "Sound waves in deformed perfectly elastic materials. Acoustoelastic effect," The Journal of the Acoustical Society of America, vol. 33, pp. 216-225, 1961.

[5] D. E. Bray and R. K. Stanley, Nondestructive Evaluation: A Tool in Design, Manufacturing and Service, CRC Press, New York, NY, USA, 1997.

[6] V. M. Malhotra and N. J. Carino, Handbook on Nondestructive Testing of Concrete, CRC Press, Pittsburgh, Pa, USA, 2nd edition, 2004.

[7] A. N. Norries, "Propagation of plane waves in a prestressed elastic medium," Journal of the Acoustical, Society of America, vol. 47, pp. 1642-1643, 1983.

[8] I. Singh, D. K. Madan, and M. Gupta, "Propagation of elastic waves in prestressed media," Journal of Applied Mathematics, vol. 2010, Article ID 817680, 11 pages, 2010.

[9] O. Udegbunam, I. O. Yaman, H. Aklan, and T. Hohm, Developing a Rapid Measure of Concrete Permeability for Use in QNQC Specifications, Proceedings of the Transportation Research Board, Paper no. 990565, 1999.

[10] R. D. Woods, "Screening of surface waves in soils," ASCE Journal of Soil Mechanics and Foundations Division, vol. 94, no. 4, pp. 951-980, 1968.

[11] K. E. Graff, Wave Motion in Elastic Solids, Oxford University Press, London, UK, 1975.

[12] ACI Committee 228, "Nondestructive test methods for evaluation of concrete in structures," Tech. Rep. ACI 228.2R-98, American Concrete Institute, Farmington Hills, Mich, USA, 2004.

[13] A. O. De La Haza, A. A. Samokrutov, and P. A. Samokrutov, "Assessment of concrete structures using the Mira and Eyecon ultrasonic shear wave devices and the SAFT-C image reconstruction technique," Construction and Building Materials, vol. 38, pp. 1276-1291, 2013.

[14] A. V. Bishko, A. A. Samokrutov, and V. G. Shevaldykin, "Ultrasonic echo-pulse tomography of concrete using shear wave low-frequency phased antenna arrays," in Proceedings of the 17th World Conference on Nondestructive Testing, Shanghai, China, 2008.

[15] R. Jones, Non-Destructive Testing of Concrete, Cambridge University Press, London, UK, 1962.

[16] T. Oh, Defect characterization in concrete element using vibration analysis of contactless impact-echo test data [Ph.D. thesis], University of Illinois, 2007.

[17] A. A. Samokrutov, V. N. Kozlov, and V. G. Shevaldykin, "Ultrasonic low-frequency transducers with dry dot contact and their applications for evaluation of concrete structures," in Proceedings of the 8th European Conference on Nondestructive Testing Conference (ECNDT '02), Barcelona, Spain, June 2002.

[18] M. Sansalone, J.-M. Lin, and W. B. Streett, "A procedure for determining $\mathrm{P}$-wave speed in concrete for use in impact-echo testing using a P-wave speed measurement technique," ACI Materials Journal, vol. 94, no. 6, pp. 531-539, 1997.

[19] J. S. Popovics, W. Song, J. D. Achenbach, J. H. Lee, and R. F. Andre, "One-sided stress wave velocity measurement in concrete," ASCE Journal of Engineering Mechanics, vol. 124, no. 12, pp. 1346-1353, 1998.

[20] C. B. Park, R. D. Miller, and J. Xia, "Multichannel analysis of surface waves," Geophysics, vol. 64, no. 3, pp. 800-808, 1999.

[21] N. Ryden, C. B. Park, P. Ulriksen, and R. D. Miller, "Multimodal approach to seismic pavement testing," Journal of Geotechnical and Geoenvironmental Engineering, vol. 130, no. 6, pp. 636-645, 2004.
[22] N. Ryden and C. B. Park, "Surface waves in inversely dispersive media," Near Surface Geophysics, vol. 2, no. 4, pp. 187-197, 2004.

[23] American Concrete Institute, "Standard test method for compressive strength of cylindrical concrete specimens," ACI Committee 39, American Concrete Institute, Farmington Hills, Mich, USA, 2014.

[24] ACI Committee, "Standard test method for static modulus of elasticity and poisson's ratio of concrete in compression," ACI Committee 469, American Concrete Institute, Farmington Hills, Mich, USA, 2014.

[25] F. D. Lydon and R. V. Balendran, "Some observations on elastic properties of plain concrete," Cement and Concrete Research, vol. 16, no. 3, pp. 314-324, 1986.

[26] British Standards Institute, "Recommendations for measurement of velocity of ultrasonic pulses in concrete," BS 1881-203, British Standards Institute, 1987.

[27] ACI Committee 214R, "Guide for evaluation of strength test results of concrete," Tech. Rep., American Concrete Institute, Farmington Hills, Mich, USA, 2011.

[28] A. H. S. Ang and W. H. Tang, Probability Concepts in Engineeirng Planning and Design, John Wiley \& Sons, New York, NY, USA, 1975. 

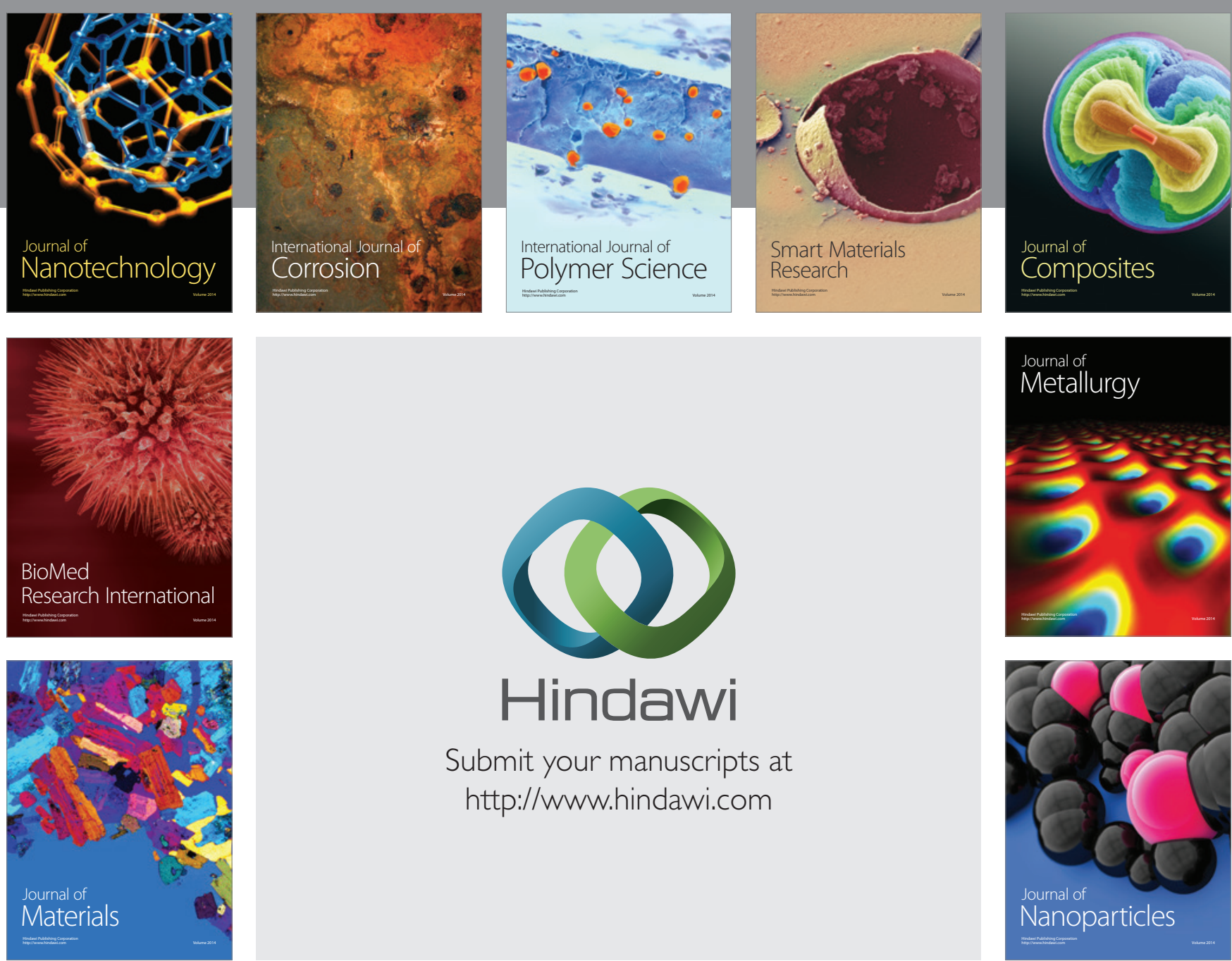

\section{Hindawi}

Submit your manuscripts at

http://www.hindawi.com

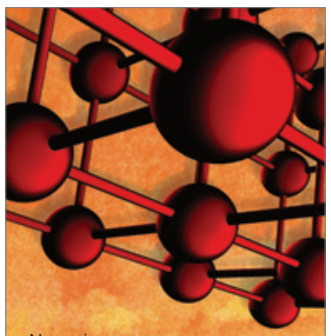

Materials Science and Engineering
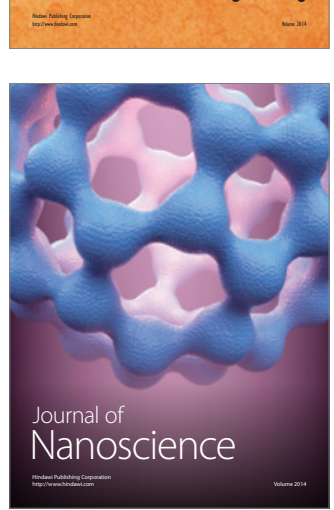
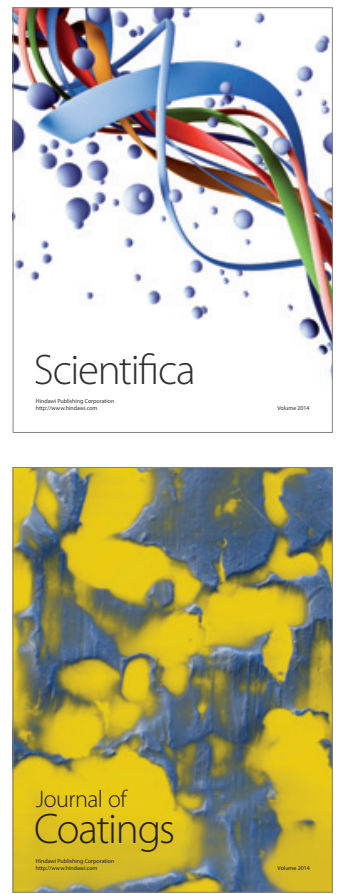
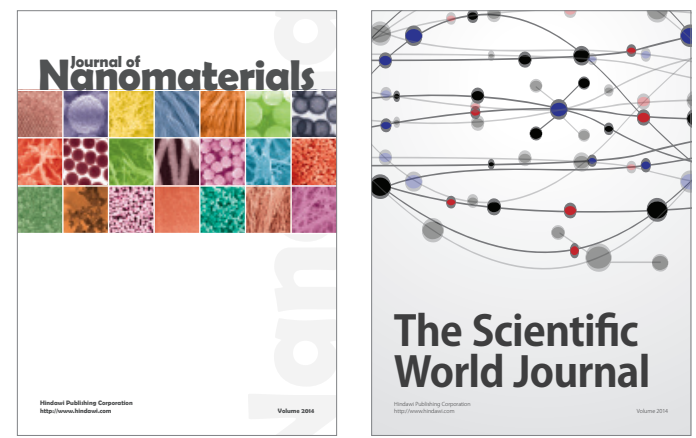

The Scientific World Journal
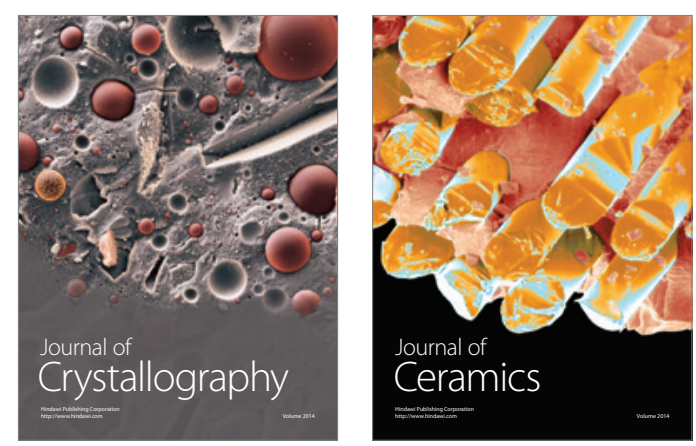
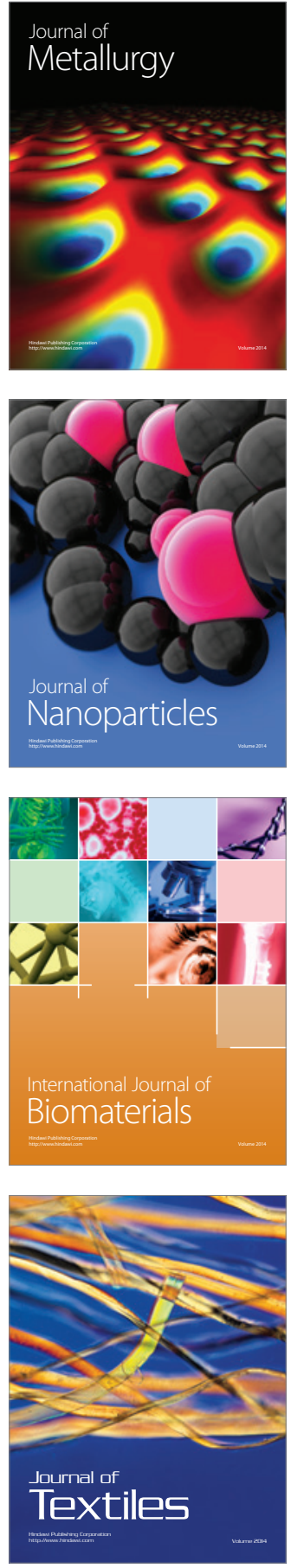